BMJ Open

Diabetes

Research

\& Care

\section{Plasma lipidomics profile in pregnancy and gestational diabetes risk: a prospective study in a multiracial/ ethnic cohort}

To cite: Rahman ML, Feng Y-CA, Fiehn 0, et al. Plasma lipidomics profile in pregnancy and gestational diabetes risk: a prospective study in a multiracial/ethnic cohort. BMJ Open Diab Res Care 2021;9:e001551. doi:10.1136/ bmjdrc-2020-001551

- Supplemental material is published online only. To view, please visit the journal online (http://dx.doi.org/10.1136/ bmjdrc-2020-001551).

MLR and Y-CAF contributed equally.

Received 13 May 2020 Revised 17 November 2020 Accepted 29 November 2020

Check for updates

(C) Where applicable, author(s) (or their employer(s)) 2021. Re-use permitted under [CC BY]. Published by BMJ

For numbered affiliations see end of article.

Correspondence to Dr Cuilin Zhang; zhangcu@mail.nih.gov and Liming Liang;

lliang@hsph.harvard.edu

\section{ABSTRACT}

Introduction Disruption of lipid metabolism is implicated in gestational diabetes (GDM). However, prospective studies on lipidomics and GDM risk in race/ethnically diverse populations are sparse. Here, we aimed to (1) identify lipid networks in early pregnancy to mid-pregnancy that are associated with subsequent GDM risk and (2) examine the associations of lipid networks with glycemic biomarkers to understand the underlying mechanisms. Research design and methods This study included 107 GDM cases confirmed using the Carpenter and Coustan criteria and 214 non-GDM matched controls from the National Institute of Child Health and Human Development Fetal Growth Studies-Singleton cohort, untargeted lipidomics data of 420 metabolites (328 annotated and 92 unannotated), and information on glycemic biomarkers in maternal plasma at visit 0 (10-14 weeks) and visit 1 (15-26 weeks). We constructed lipid networks using weighted correlation network analysis technique. We examined prospective associations of lipid networks and individual lipids with GDM risk using linear mixed effect models. Furthermore, we calculated Pearson's partial correlation for GDM-related lipid networks and individual lipids with plasma glucose, insulin, C-peptide and glycated hemoglobin at both study visits. Results Lipid networks primarily characterized by elevated plasma diglycerides and short, saturated/low unsaturated triglycerides and lower plasma cholesteryl esters, sphingomyelins and phosphatidylcholines were associated with higher risk of developing GDM (false discovery rate (FDR) <0.05). Among individual lipids, 58 metabolites at visit 0 and 96 metabolites at visit 1 (40 metabolites at both time points) significantly differed between women who developed GDM and who did not (FDR <0.05). Furthermore, GDM-related lipid networks and individual lipids showed consistent correlations with maternal glycemic markers particularly in early pregnancy at visit 0 .

Conclusions Plasma lipid metabolites in early pregnancy both individually and interactively in distinct networks were associated with subsequent GDM risk in race/ethnically diverse US women. Future research is warranted to assess lipid metabolites as etiologic markers of GDM.

\section{INTRODUCTION}

Gestational diabetes (GDM) is one of the most common pregnancy complications and has

\section{Significance of this study}

What is already known about this subject?

- Emerging epidemiological studies in non-pregnant population have identified lipid metabolites prospectively associated with insulin resistance and type 2 diabetes; however, prospective studies among pregnant women on lipidomic profiles and gestational diabetes (GDM) are sparse.

- We examined plasma lipidomic profile in early pregnancy to mid-pregnancy in relation to the subsequent risk of GDM in a multiracial/ethnic US population.

What are the new findings?

- We identified a list of glycerolipids and some glycerophospholipids, sterol lipids and sphingomyelins measured in maternal plasma as early as in 10-14 weeks of gestation individually and interactively in distinct metabolite networks were associated with GDM risk.

These metabolites and their interactive networks also showed correlation patterns with maternal nonfasting and fasting glycemic biomarkers that were in consistent with findings on GDM.

How might these results change the focus of research or clinical practice?

- This study sheds light on the importance of lowmolecular-weight intermediate lipid metabolites as etiological biomarkers of GDM.

been linked with both short-term and longterm adverse health consequences for the woman and her child. For instance, women diagnosed with GDM are at 4.8-11.5 times higher risk of developing type 2 diabetes later in life. ${ }^{1}$ Their children are more likely to be macrosomic at birth and are at a higher risk of developing childhood obesity and glucose intolerance during adulthood. ${ }^{2}{ }^{3}$ Therefore, identifying etiological biomarkers and modifiable risk factors of GDM are important 
milestones to design effective intervention strategy and improve the health and well-being of both women and their children. ${ }^{4}$

Prior studies point to disturbances in lipid metabolism in developing glucose homeostasis, ${ }^{5}$ however the traditional lipid biomarkers such as total triglycerides and cholesterol do not reflect the complexity of the altered lipid metabolism associated with GDM. Lipidomics is an emerging tool of quantitative analysis of the full spectra of low-molecular-weight intermediate lipid metabolites, which may reflect a snapshot of the dynamic biochemical activities and provide new insights into the underlying etiology of GDM. ${ }^{67}$ A longitudinal evaluation of the lipidome in early pregnancy to mid-pregnancy can also capture the temporal variation in lipid profiles, accounting for exogenous (diet) and endogenous lipid metabolism.

Emerging studies among non-pregnant individuals have identified novel lipid biomarkers prospectively associated with insulin resistance, ${ }^{8}$ type 2 diabetes $^{9}$ and transition to type 2 diabetes from GDM. ${ }^{10}{ }^{11}$ Only two studies have prospectively evaluated the lipidome with GDM risk. ${ }^{12} 13$ However, those studies were conducted exclusively among white Caucasian or Han Chinese populations, limiting the generalisability of findings to other populations. Inference from these studies were also hindered by small sample size $(\text { GDM cases }=21)^{12}$ or liability for residual confounding because of a lack of information on conventional risk factors, such as family history of diabetes. ${ }^{13}$ To address these critical research gaps, we aimed to (1) prospectively evaluate the associations of maternal plasma lipidomics profile at two-time windows before GDM diagnosis (ie, in early pregnancy and mid-pregnancy) with the subsequent risk of developing GDM in a race/ethnically diverse US population. We also aimed to examine the associations of lipidomics profile with maternal glycemic biomarkers to understand the etiologic role of lipid metabolites in the development of GDM.

\section{SUBJECTS AND METHODS}

\section{Study population and design}

We conducted a case-control study nested within the Eunice Kennedy Shriver National Institute of Child Health and Human Development (NICHD) Fetal Growth Studies-Singletons cohort, which is a multicenter, multiracial/ethnic prospective pregnancy cohort. During 20092013, 2802 pregnant women aged 18-40 years without pre-existing hypertension, diabetes, cancer and other chronic diseases were recruited between 8 and 13 weeks of pregnancy through 12 clinical centers across the USA. The detail of the study has been previously described. ${ }^{14}$

On recruitment at 8-13 weeks (visit 0 ), women were scheduled to attend five in-hospital follow-up visits at targeted gestational weeks: 16-22 (visit 1), 24-29 (visit 2), 30-33 (visit 3), 34-37 (visit 4) and 38-41 (visit 5). Venous blood samples were collected at visits $0,1,2$ and 4 , where only visit 1 samples were collected after an overnight fast. Because some women arrived late for their scheduled visit, the actual blood collection windows ranged slightly beyond the targeted time windows (ie, weeks 10-14 (visit 0), 15-26 (visit 1), 23-31 (visit 2), 33-39 (visit 4)), the first two of which were before GDM diagnosis. Plasma samples were processed immediately after blood collection and stored at $-80^{\circ} \mathrm{C}$ until analysis.

Within the cohort, we identified 107 incident GDM cases based on the Carpenter and Coustan criteria through medical records review of oral glucose tolerance test (OGTT) results following the American College of Obstetrics and Gynecologists recommendations (online supplemental figure S1). ${ }^{15} 16$ OGTT was performed at a mean $( \pm \mathrm{SD})$ gestational age of $27.5( \pm 4.3)$ weeks. We also selected 214 controls matched with the cases in a 2:1 ratio on age ( \pm 2 years), self-reported race/ethnicity and gestational week of blood collection ( \pm 2 weeks). Of note, the majority of controls $(n=195)$ were screened for GDM using the $50 \mathrm{~g}$ glucose challenge test. For those without routine GDM screening $(\mathrm{n}=19), 12$ went through an OGTT with normal glucose values below the Carpenter and Coustan criteria thresholds and the remaining were free of hospital discharge diagnosis of GDM.

\section{Laboratory assays}

Lipidome analysis was performed at the West Coast Metabolomics Center, University of California Davis Genome Center. Details of laboratory methods used to quantify lipids were presented elsewhere. ${ }^{17}$ Briefly, plasma lipidome was quantified using high-throughput liquid chromatography quadruple time-of-flight mass spectrometry (LC-QTOF MS/MS) ${ }^{18}{ }^{19}$ Internal standards were used for the calibration of retention times. ${ }^{17}$ Signals across samples were corrected by a bioreclamation quality control plasma-based normalization method.

A total of 420 non-targeted lipid metabolites, including 328 annotated and 92 unannotated metabolites were profiled at visits $0,1,2$ and 4 . The annotated metabolites belonged to four major lipid categories: glycerolipids $(n=145)$, including monoglycerides ( $M G, n=3)$, diglycerides (DG, $\mathrm{n}=16$ ), triglycerides (TG, $\mathrm{n}=126)$; glycerophospholipids $(\mathrm{n}=116)$, including phosphatidylcholines ( $\mathrm{PC}, \mathrm{n}=83)$, phosphatidylethanolamines ( $\mathrm{PE}$, $\mathrm{n}=11$ ) and lysophosphatidylcholines (LPC, $\mathrm{n}=22)$; sphingolipids $(n=62)$, including sphingomyelins $(S M, n=34)$, ceramides (Cer, $n=15$ ), glucosylceramides (GlcCer, $n=9$ ), lactosylceramides (LacCer, $n=4)$ and sterol lipids $(n=20)$, primarily cholesteryl esters $(\mathrm{CE}, \mathrm{n}=18$ ).

We also measured a panel of glycemic biomarkers in maternal plasma at the same study visits. Concentrations of glucose, insulin and C-peptide were measured using hexokinase, immunosorbent, sandwich immunoassay and immunoturbidimetric assays (Roche Diagnostics, Indianapolis, Indiana), respectively. Glycated hemoglobin (HbA1c) was measured in whole blood using chromatography (Tosoh Bioscience, California and Tokyo, Japan). All assays had inter-assay and intra-assay coefficients of 
variation $<10 \%$ and were performed without the knowledge of GDM status.

\section{Covariates}

Data on maternal demographic, lifestyle and clinical factors were collected at each visit using a standardized and structured questionnaire. Potential confounders of exposure-outcome relationship were selected a priori based on causal diagram, ${ }^{20}$ which included maternal age (continuous), family history of diabetes (yes, no), enrollment body mass index (BMI) (continuous), alcohol use before pregnancy (yes, no), race/ethnicity (white, nonHispanic black, Hispanic and Asians) and gestational age at blood collection (continuous). We also collected information on GDM treatment (intervention using medication, intervention using diet or lifestyle modification but no medication and no intervention undertaken) from medical records. In this low-risk population, women without obesity who smoked 6 months preceding the index pregnancy were ineligible, and only five women with obesity reported smoking during the same period before pregnancy. Thus, in our main analysis, we did not include smoking as a covariate.

\section{Statistical analysis}

All analyses were conducted in R V.3.5.2 (Austria, Vienna) and SAS V.9.4 (SAS Institute, Cary, North Carolina, USA). The bivariate characteristics of GDM cases and non-GDM controls at baseline were compared using linear mixed models, accounting for matched case-control pairs. For associations with GDM risk, lipidome data collected prior to GDM diagnosis at visit 0 (10-14 weeks) and visit 1 (15-26 weeks) were used in the analysis to preserve the temporal relation.

\section{Metabolite coregulating network analysis}

We applied weighted correlation network analysis ( $\mathrm{R}$ package) to construct networks of highly correlated lipid metabolites, and examined how metabolites within an interconnecting network collectively influence GDM risk. ${ }^{21}$ Lipidomics data were normalized using quantile normalization to reduce batch effects and then standardized using inverse-normal transformation to remove the effect of potential outliers. To construct metabolite networks, first we obtained network adjacency matrix, ${ }^{22}$ a measure of connectedness between each pair of metabolites, estimated by Pearson's correlation coefficients. We then transformed network adjacency matrix to topological overlap matrix (TOM), ${ }^{23}$ a measure of connectedness between each pair of metabolites in a network considering their relation to all other metabolites within that network and subsequently performed hierarchical clustering on TOM-based dissimilarity. Consecutively, we applied the Dynamic Tree Cut function ${ }^{24}$ to identify network clusters consisting of highly correlated metabolites that follow a scale-free topology, which is a type of network configuration characterized by a few metabolites having many connections with neighboring metabolites but most metabolites having just a handful of connections. ${ }^{25}$ This analytical approach was adopted based on reports suggesting that most metabolic networks in a biological system follow a scale-free topology. ${ }^{25}$

To evaluate associations between lipid networks and GDM risk, we used the first eigenvector of each identified network in linear mixed-effect models, where metabolite network score was used as the response variable and GDM status as the independent variable, adjusting for age, gestational week at blood collection, enrollment BMI, self-reported race/ethnicity, alcohol use before pregnancy and family history of diabetes. Case-control pair ID was modeled as random intercept to account for correlation within matched pair. Multiple testing was corrected using false discovery rate (FDR). To identify individual metabolites within a network that were driving the associations, we estimated network membership score, a measure of importance of member metabolites within each network, as the correlation between metabolite concentrations and the first eigenvector of respective metabolite network.

Next, we examined correlations between metabolite networks and maternal glycemic markers using Pearson's partial correlations adjusting for covariates. Participants missing information on insulin $(n=1)$, glucose $(n=6)$ and HbAlc $(n=6)$ at visit 0 and fasting insulin, $(n=2)$, fasting C-peptide $(n=2)$, fasting glucose $(n=2)$ and fasting $\operatorname{HbA1c}(n=2)$ at visit 1 were excluded from the analyses. Concentration of glycemic markers was natural log transformed prior to analyses. We applied inverse probability weighting to account for the matched case-control design.

\section{Individual metabolite analysis}

We also examined individual metabolites, one at a time, in relation to GDM using similar mixed effect models, adjusting for covariates. First, we normalized the data using inverse-normal transformation within batches. We analyzed the data separately by batch and then combined results using inverse-variance weighted meta-analysis to minimize batch effect. Analyses were corrected for multiple testing using FDR. We visualized results in a twodimensional space by the number of acyl chain carbon atoms (x-axis) and double bond contents (y-axis) of individual metabolites within each lipid class because prior studies reported that the number of acyl carbons and double bonds were important characteristics to determine glycemic risk of endogenous metabolites. ${ }^{26}$ Plots were visually inspected for patterns of association by acyl carbon chain length and double bond contents.

Next, we estimated correlations between individual metabolites with maternal glycemic biomarkers using Pearson's partial correlation, adjusting for covariates. Finally, leveraging on repetitive measures of lipidomics data, we examined the mean abundance of selected metabolites between GDM cases and controls across pregnancy to assess temporal trend of selected metabolites with GDM status. The case-control difference in metabolite concentrations was assessed using the two-sample 
Table 1 Participant characteristics among women with gestational diabetes (GDM) and their matched* controls: the NICHD Fetal Growth Studies-Singleton Cohort

\begin{tabular}{|c|c|c|c|c|}
\hline Characteristics & $\mathbf{N}$ & GDM cases $(n=107)$ & Non-GDM controls $(n=214)$ & $P$ value \\
\hline Age, years & 321 & $30.4 \pm 5.7$ & $30.5 \pm 5.4$ & \\
\hline Race/Ethnicity & 321 & & & \\
\hline Non-Hispanic white & & $25(23.4 \%)$ & $50(23.4 \%)$ & \\
\hline Non-Hispanic black & & $15(14.0 \%)$ & $30(14.0 \%)$ & \\
\hline Hispanic & & $41(38.3 \%)$ & $82(38.3 \%)$ & \\
\hline Asian/Pacific Islander & & $26(24.3 \%)$ & $52(24.3 \%)$ & \\
\hline Enrollment BMI, kg/m² & 321 & $27.8 \pm 6.2$ & $25.6 \pm 5.3$ & 0.0005 \\
\hline Family history of diabetes & 321 & $40(37.4 \%)$ & $48(22.4 \%)$ & 0.003 \\
\hline Alcohol use before pregnancy & 321 & $61(57.0 \%)$ & $137(64.0 \%)$ & 0.22 \\
\hline Smoking before pregnancy & 321 & $4(3.7 \%)$ & $1(0.5 \%)$ & 0.02 \\
\hline Fasting glucose $\dagger, \mathrm{mg} / \mathrm{dL}$ & 313 & $91.92 \pm 14.6$ & $83.38 \pm 7.9$ & $<0.001$ \\
\hline Fasting insulint, pmol/L & 313 & $135.41 \pm 204.6$ & $66.87 \pm 95.2$ & $<0.001$ \\
\hline Fasting C-peptide†, nmol/L & 313 & $0.92 \pm 0.7$ & $0.60 \pm 0.4$ & $<0.001$ \\
\hline Fasting $\mathrm{HbA} 1 \mathrm{c}+, \%$ & 313 & $5.18 \pm 0.5$ & $4.95 \pm 0.3$ & 0.002 \\
\hline
\end{tabular}

Values are $\mathrm{n}(\%)$ or mean \pm SD.

$P$ values were not shown for matching variables: age and race/ethnicity.

*Matching factors: age ( \pm 2 years), race/ethnicity (non-Hispanic white, non-Hispanic black, Hispanic or Asian/Pacific Islander) and the gestational week of blood collection ( \pm 2 weeks).

†Glycemic biomarkers were measured in fasting samples at 15-26 weeks (visit 1). Sample size for fasting glucose was 98 cases and 213 controls and for fasting insulin, C-peptide and HbA1c was 99 cases and 212 controls. Values were log transformed before fitting into linear mixed effect models.

BMI, body mass index; HbA1c, glycated hemoglobin; NICHD, National Institute of Child Health and Human Development.

t-tests. SEs of the mean and $p$ values were also plotted at each specific time point.

\section{Sensitivity analysis}

For associations between lipid networks and GDM risk, we conducted sensitivity analysis additionally adjusting for maternal smoking status prior to pregnancy to assess residual confounding of our results by maternal smoking status. To examine potential reverse causation by GDM severity status, we conducted a sensitivity analysis stratified by GDM treatment group (intervention using medication; intervention using diet or lifestyle modification but no medication and no intervention undertaken) at both study visits.

\section{RESULTS}

Women who developed GDM ( $\mathrm{n}=107)$ were more likely to have higher BMI at the time of enrollment and a positive family history of diabetes compared with non-GDM controls $(\mathrm{n}=214)$ (table 1). GDM women also had higher concentrations of fasting glucose, fasting insulin, C-peptide and $\mathrm{HbAlc}$ at visit 0.

\section{Lipid coregulating networks and GDM risk}

We identified eight coregulating lipid networks at visit 0 and six networks at visit 1 ; the networks were in general well preserved between study visits (figure 1). In both visits, the 'yellow' and 'brown' networks, which primarily comprised short, saturated/low unsaturated TGs were positively associated with GDM risk, whereas the 'turquoise' network primarily comprised CEs, PCs and some long, polyunsaturated TGs, and the 'blue' network primarily comprised SMs and ceramides were negatively associated with GDM risk (FDR $<0.05$ ).

\section{Lipid coregulating networks and glycemic biomarkers}

GDM-related lipid networks showed moderate to weak but largely consistent correlations with glycemic biomarkers, although the strength of association differed by study visits (figure 2). In early pregnancy at visit 0 , cross-sectional analyses showed that the 'turquoise' and 'blue' networks were negatively correlated with nonfasting plasma insulin, C-peptide and $\mathrm{HbAlc}$, whereas the 'brown' and 'yellow' networks showed the opposite pattern. Similar but weaker correlation pattern was observed in prospective analyses of metabolite networks at visit 0 with fasting glycemic markers measured at visit 1. Specifically, the 'turquoise' network at visit 0 was negatively correlated with $\mathrm{HbA1c}(\mathrm{r}=-0.16, \mathrm{p}=0.009)$ at visit 1 , whereas the 'yellow' network at visit 0 was positively correlated with fasting glucose $(\mathrm{r}=0.14, \mathrm{p}=0.02)$, insulin $(\mathrm{r}=0.12, \mathrm{p}=0.05)$ and $\mathrm{C}$-peptide $(\mathrm{r}=0.18, \mathrm{p}=0.002)$ at visit 1. Cross-sectional correlations between GDM-related lipid networks and fasting glycemic biomarkers at visit 1 were weaker but showed a consistent pattern.

\section{Individual lipid metabolites with GDM risk}

Table 2 presents associations of selected individual metabolites with GDM risk by study visit (complete results are 
Visit 0 (10 - 14 weeks)
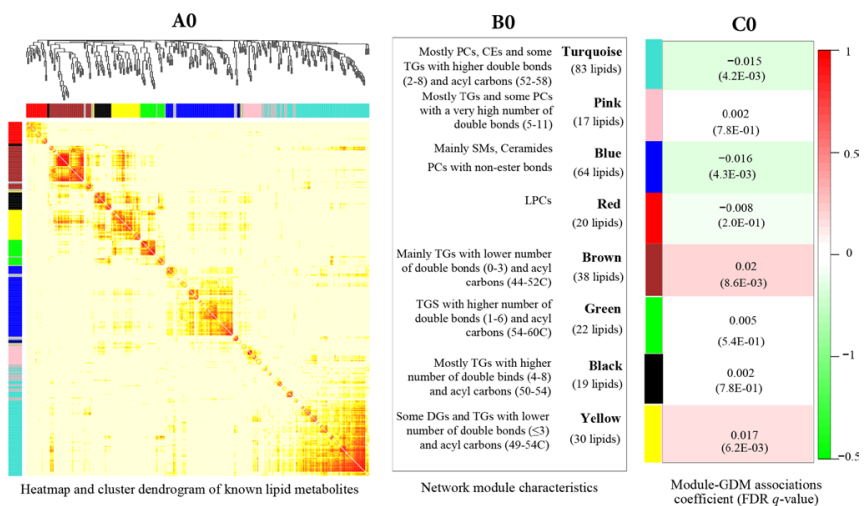

Visit 1 (15 - 26 weeks)
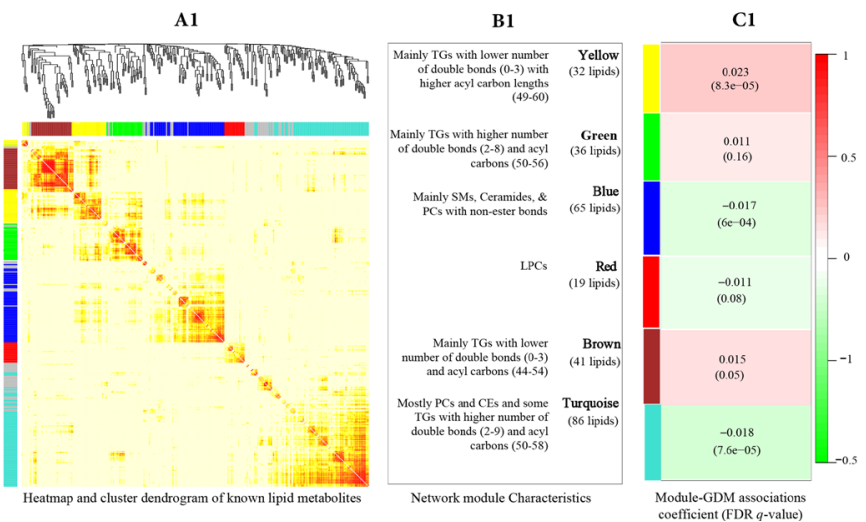

Figure 1 Lipid networks represented by correlated metabolites, as shown in cluster dendrogram and heatmap at visit $0(\mathrm{~A} 0)$ and visit 1 (A1). We constructed eight lipid networks at visit 0 (B0) and six networks at visit 1 (B1) from 328 annotated lipid metabolites using weighted correlation network analysis algorithm. Constituents of each lipid network are presented. The associations between lipid networks and GDM risk (coefficient (FDR)) using linear mixed effect models, adjusted for maternal age, enrollment BMI, family history of diabetes, alcohol use before pregnancy, race/ethnicity and gestational age at blood collection at visit 0 (C0) and visit 1 (C1) are presented. BMI, body mass index; CE, cholesteryl ester; FDR, false discovery rate; GDM, gestational diabetes; LPC, lysophosphatidylcholine; PC, phosphatidylcholine; SM, sphingomyelin; TG, triglyceride.

presented in online supplemental table 1). Specifically, 58 metabolites (47 annotated, 11 unannotated) at visit 0 and 96 metabolites (75 annotated and 21 unannotated) at visit 1 (40 metabolites at both study visits) were significantly associated with GDM risk (FDR $<0.05)$. At visit 0 , higher plasma concentrations of 41 metabolites (6 DGs, 30 TGs, 4 PCs and $1 \mathrm{PE}$ ) and lower concentration of 6 metabolites (all CEs) were associated with higher risk of GDM. For instance, TG $\left(50: 1 ; 10.80 \_850.79\right)$, a member of the 'brown' network, showed the most significant positive association with GDM $(\beta=0.54 ; \mathrm{FDR}=0.0003)$ at visit 0 , whereas CE (18:2; 10.37_671.57), a member of the 'turquoise' network showed the most significant negative association $(\beta=-0.52 ; \mathrm{FDR}=0.001)$. Similar associations were observed at visit 1 . For instance, higher plasma

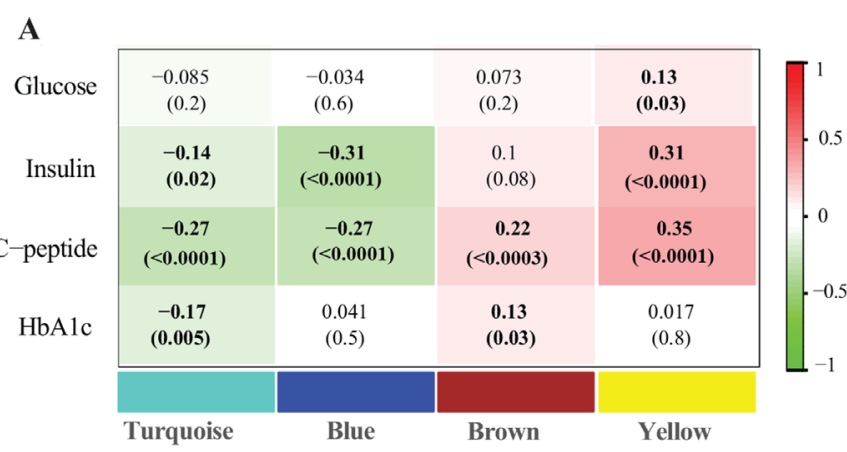

\section{B}
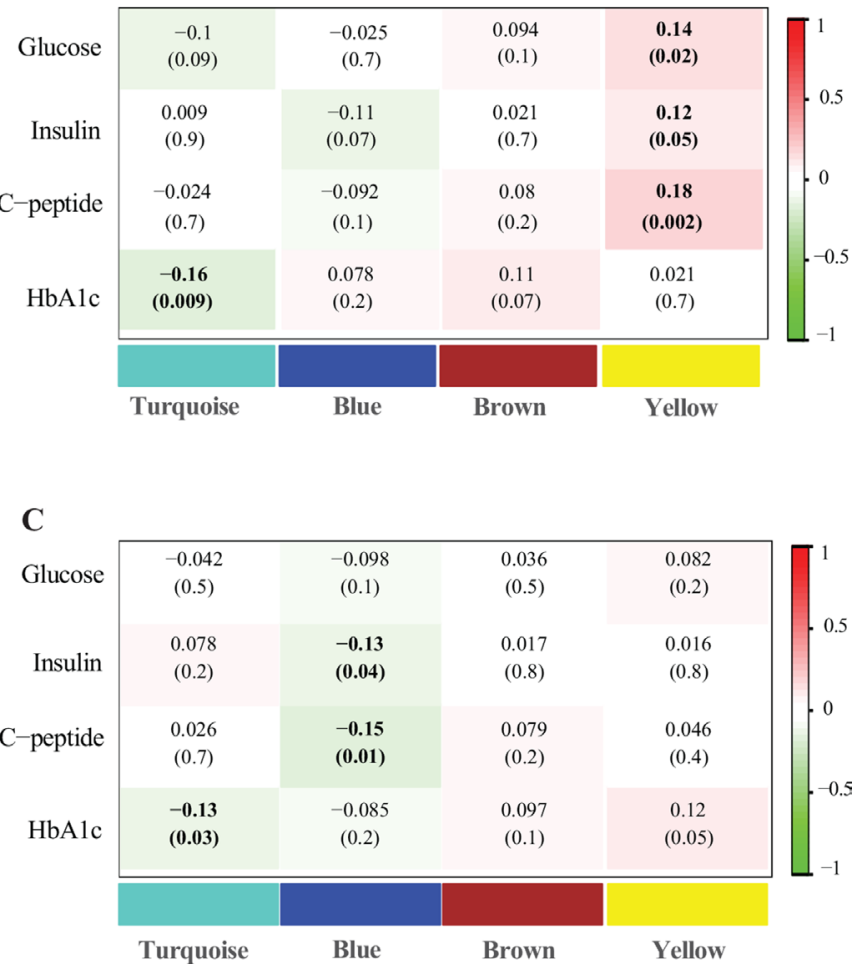

Figure 2 Pearson's partial correlation coefficients ( $p$ values) between GDM-related lipid networks and maternal glycemic markers, adjusted for maternal age, enrollment BMI, family history of diabetes, alcohol use before pregnancy, race/ ethnicity and gestational age at blood collection. (A) Crosssectional associations of lipid networks with non-fasting glycemic markers at visit 0; (B) prospective associations of lipid networks at visit 0 with fasting glycemic markers at visit 1; (C) cross-sectional associations of lipid networks and fasting glycemic markers at visit 1 . BMI, body mass index; GDM, gestational diabetes; HbA1c, glycated hemoglobin.

concentrations of 64 metabolites (6 GDs, 52 TGs, 3 PEs, $1 \mathrm{PC}$ and $2 \mathrm{SMs}$ ) and lower concentrations of 14 metabolites (6 CEs, 4 PCs, 1 LPC and $3 \mathrm{SMs}$ ) at visit 1 were associated with higher risk of GDM. Again, TG (50:1; $10.80 \_850.79$ ) showed the most significant positive association with GDM $(\beta=0.51, \mathrm{FDR}=0.0006)$ at visit 1 , whereas CE (18:1; 10.85_673.59), a member of the 'turquoise' network, showed the most significant negative association $(\beta=-0.53, \mathrm{FDR}=0.004)$. 
Table 2 Selected individual metabolites and their associations with GDM risk by study visit

\begin{tabular}{|c|c|c|c|c|c|c|c|c|}
\hline \multirow[b]{2}{*}{ Metabolite } & \multirow[b]{2}{*}{ RT_m/z } & \multirow[b]{2}{*}{$\begin{array}{l}\text { Corresponding } \\
\text { lipid network }\end{array}$} & \multicolumn{3}{|c|}{ Visit 0 (10-14 weeks) } & \multicolumn{3}{|c|}{ Visit 1 (15-26 weeks) } \\
\hline & & & $\begin{array}{l}\text { Network } \\
\text { membership } \\
\text { score* }^{*}\end{array}$ & Estimate & FDR & $\begin{array}{l}\text { Network } \\
\text { membership } \\
\text { score* }^{*}\end{array}$ & Estimate & FDR \\
\hline TG (50:1) & 10.80_850.79 & Brown & 0.80 & 0.54 & 0.0003 & 0.80 & 0.51 & 0.0006 \\
\hline TG (50:2) & 10.41_853.73 & Brown & 0.55 & 0.55 & 0.0003 & 0.64 & 0.43 & 0.0037 \\
\hline TG (50:2) & 10.41_848.77 & Brown & 0.73 & 0.54 & 0.0003 & 0.73 & 0.43 & 0.0037 \\
\hline TG (54:5) & 10.32_903.74 & & & 0.50 & 0.0011 & & 0.44 & 0.0043 \\
\hline CE (18:2) & 10.37_671.57 & Turquoise & 0.95 & -0.52 & 0.0011 & 0.94 & -0.33 & 0.0290 \\
\hline Unannotated & 10.80_551.50 & & & 0.50 & 0.0011 & & 0.51 & 0.0006 \\
\hline TG (48:1) & 10.38_822.75 & Brown & 0.93 & 0.48 & 0.0016 & 0.92 & 0.42 & 0.0049 \\
\hline Unannotated & 10.39_549.49 & & & 0.47 & 0.0016 & & 0.37 & 0.0097 \\
\hline TG (48:1) & 10.38_827.71 & Brown & 0.93 & 0.48 & 0.0017 & 0.94 & 0.36 & 0.0170 \\
\hline TG (50:1) & 10.80_855.74 & Brown & 0.51 & 0.47 & 0.0029 & 0.53 & 0.41 & 0.0059 \\
\hline DG (34:1) & 6.85_612.56 & Yellow & 0.83 & 0.44 & 0.0048 & 0.75 & 0.45 & 0.0031 \\
\hline TG (52:1) & 11.20_878.82 & Yellow & 0.81 & 0.43 & 0.0054 & 0.80 & 0.50 & 0.0011 \\
\hline PC (38:3) & 5.79_812.61 & & & 0.42 & 0.0054 & & 0.36 & 0.0200 \\
\hline Unannotated & 10.80_577.52 & & & 0.42 & 0.0054 & & 0.44 & 0.0021 \\
\hline TG (48:2) & $9.98 \_820.74$ & Brown & 0.84 & 0.43 & 0.0054 & 0.88 & 0.34 & 0.0230 \\
\hline TG (50:3) & 10.01_846.76 & Yellow & 0.80 & 0.42 & 0.0054 & 0.76 & 0.41 & 0.0046 \\
\hline TG (52:1) & 11.20_883.77 & Brown & 0.57 & 0.42 & 0.0059 & 0.63 & 0.48 & 0.0016 \\
\hline TG (58:6) & 10.65_957.79 & & & 0.44 & 0.0059 & & 0.43 & 0.0047 \\
\hline DG (34:1) & 6.85_617.51 & Yellow & 0.67 & 0.42 & 0.0059 & 0.46 & 0.43 & 0.0037 \\
\hline Unannotated & 11.20_899.75 & & & 0.40 & 0.0064 & & 0.40 & 0.0047 \\
\hline TG (48:0) & 10.81_824.77 & Brown & 0.78 & 0.41 & 0.0072 & 0.82 & 0.52 & 0.0008 \\
\hline TG (58:6) & 10.65_952.83 & Yellow & 0.68 & 0.41 & 0.0117 & 0.71 & 0.44 & 0.0037 \\
\hline CE (18:1) & 10.85_673.59 & Turquoise & 0.89 & -0.40 & 0.0133 & 0.88 & -0.53 & 0.0004 \\
\hline TG (46:0) & 10.36_796.74 & Brown & 0.93 & 0.37 & 0.0165 & 0.96 & 0.41 & 0.0073 \\
\hline Unannotated & 6.39_615.49 & & & 0.36 & 0.0197 & & 0.47 & 0.0016 \\
\hline Unannotated & 10.31_951.81 & & & 0.37 & 0.0204 & & 0.39 & 0.0071 \\
\hline TG (51:1) & 10.93_869.76 & Yellow & 0.80 & 0.37 & 0.0221 & 0.76 & 0.36 & 0.0120 \\
\hline TG (51:1) & 10.93_864.80 & Yellow & 0.90 & 0.36 & 0.0221 & 0.84 & 0.36 & 0.0120 \\
\hline CE (18:1) & 10.85_668.63 & Turquoise & 0.94 & -0.36 & 0.0267 & 0.92 & -0.40 & 0.0047 \\
\hline TG (54:2) & 11.20_904.83 & Yellow & 0.79 & 0.36 & 0.0281 & 0.82 & 0.43 & 0.0047 \\
\hline TG $(54: 1)$ & 11.56_906.85 & Yellow & 0.77 & 0.35 & 0.0328 & 0.86 & 0.45 & 0.0042 \\
\hline CE (22:6) & 9.88_719.57 & Turquoise & 0.94 & -0.34 & 0.0328 & 0.94 & -0.34 & 0.0250 \\
\hline TG (56:4) & 10.88_933.79 & & & 0.33 & 0.0388 & & 0.40 & 0.0049 \\
\hline TG (49:1) & 10.59_836.77 & Yellow & 0.80 & 0.32 & 0.0415 & 0.74 & 0.28 & 0.0470 \\
\hline Unannotated & 10.17_896.79 & & & 0.32 & 0.0415 & & 0.33 & 0.0270 \\
\hline TG (48:0) & 10.81_829.73 & Brown & 0.67 & 0.32 & 0.0484 & 0.73 & 0.41 & 0.0071 \\
\hline
\end{tabular}

FDR q values were calculated based on 420 individual tests for 420 metabolites.

*Network membership score represents a measure of importance for member metabolites within a network and ranges between -1 and 1. Network membership scores were not presented for metabolites that failed to form a network and for unannotated metabolites.

CE, cholesteryl esters; DG, diglyceride; FDR, false discovery rate; GDM, gestational diabetes; GlcCer, glucosylceramide; LPC, lysophosphatidylcholine; PC, phosphatidylcholine; RT_m/z, retention time_mass-to-charge ratio; SM, sphingomyelin; TG, triglyceride. 

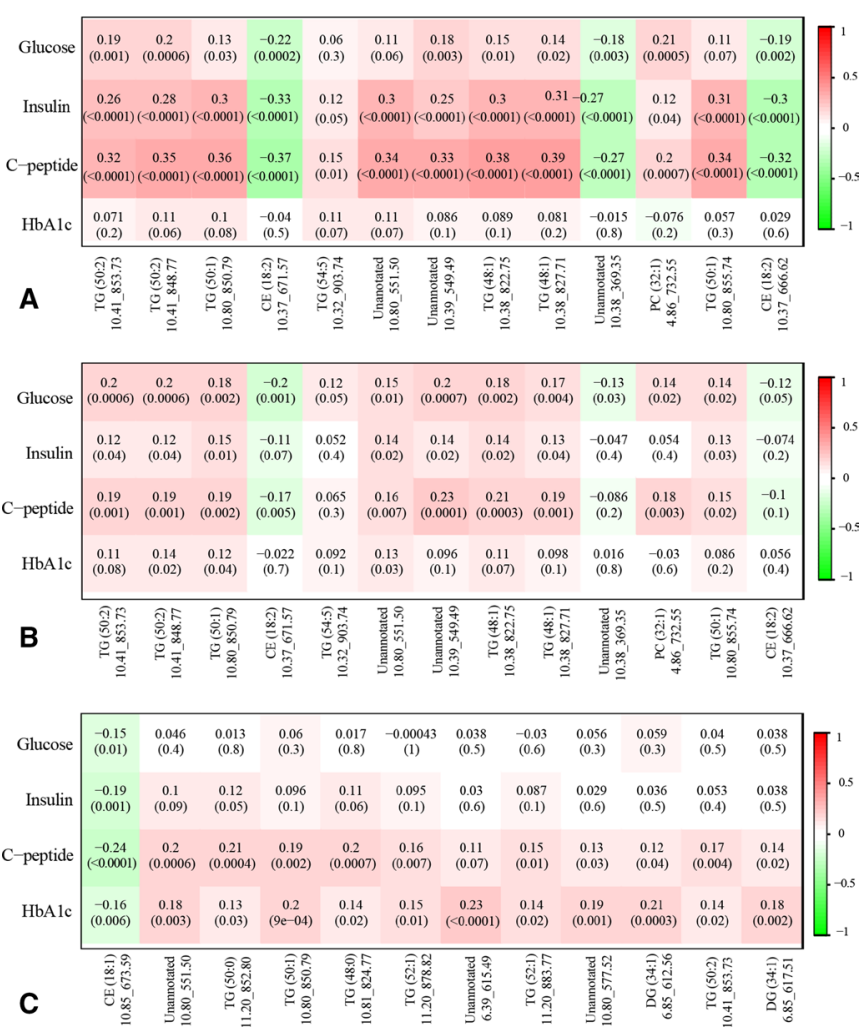

Figure 3 Pearson's partial correlation coefficients ( $p$ values) between GDM-related selected lipid metabolites with maternal glycemic markers, adjusted for maternal age, enrollment BMI, family history of diabetes, alcohol use before pregnancy, race/ethnicity and gestational age at blood collection. (A) Cross-sectional associations of selected lipid metabolites with non-fasting glycemic markers at visit 0; (B) Prospective associations of selected lipid metabolites at visit 0 with fasting glycemic markers at visit 1 ; (C) Crosssectional associations of selected lipid metabolites and fasting glycemic markers at visit 1. BMI, body mass index; $\mathrm{CE}$, cholesteryl ester; DG, diglyceride; GDM, gestational diabetes; HbA1c, glycated hemoglobin; TG, triglyceride.

\section{Individual lipid metabolite-GDM relations by acyl carbon chain length and bond characteristics}

We illustrated associations of individual lipid metabolites with GDM risk by acyl carbon chain length and double bond contents of the metabolites (online supplemental figure S2), as prior studies have identified these properties as important determinants of cardiometabolic risk. ${ }^{87}$ Visual inspection of scatter plots suggests that short, saturated or less unsaturated TGs and saturated SMs were more likely to be associated with higher risk of GDM. No clear association pattern was observed for other lipid classes.

\section{GDM-related individual lipid metabolites and glycemic biomarkers}

GDM-related individual lipid metabolites also showed consistent correlations with maternal glycemic biomarkers (figure 3). For example, plasma concentration of TG $\left(50: 1 ; 10.80 \_850.79\right)$ was significantly and positively correlated with plasma glucose $(\mathrm{r}=0.19$, $\mathrm{p}=0.001)$, insulin $(\mathrm{r}=0.26, \mathrm{p}<0.0001)$ and C-peptide $(\mathrm{r}=0.32, \mathrm{p}<0.0001)$ in cross-sectional analyses at visit 0 , whereas CE (18:2; 10.37_671.57) was significantly and negatively correlated with glucose $(r=-0.22, p<0.0002)$, insulin $(\mathrm{r}=-0.33, \mathrm{p}<0.0001)$ and C-peptide $(\mathrm{r}=-0.37$, $\mathrm{p}<0.0001)$. A similar but weaker correlation pattern was observed in prospective analyses of metabolites measured at visit 0 with fasting plasma glucose, insulin, and C-peptide measured at visit 1 . In cross-sectional analyses at visit 1 , we observed a consistent correlation pattern particularly for fasting plasma C-peptide and HbAlc.

\section{Temporal trend of GDM-related individual lipid metabolites across pregnancy}

Leveraging on longitudinally collected lipidomics data, we further examined plasma concentrations of selected GDM-related metabolites between cases and controls across pregnancy (online supplemental figure S3). Overall, the difference in mean metabolite concentration by case-control status, if any, was mostly observed before the diagnosis of GDM (24-28 weeks), which progressively attenuated in late pregnancy. For example, the mean plasma concentration of TG (50:2), TG (50:0), TG (50:1) and TG (48:0) were significantly higher among GDM cases compared with non-GDM controls at visit 0 and visit 1 , whereas the opposite pattern was observed for $\mathrm{CE}$ (18:1) and CE (18:2).

\section{Sensitivity analyses}

Sensitivity analysis additionally adjusting the models for maternal smoking prior to pregnancy did not change the associations between lipid networks and GDM risk at both study visits. Additional sensitivity analysis stratified by GDM treatment status suggested that the associations between lipid networks and GDM risk may be influenced by disease severity status as early as 10-14 weeks. Overall, we observed consistent associations for lipid networks with GDM risk among women who developed GDM but did not require medical intervention (insulin or other medication) at both study visits. For example, the 'turquoise' and 'blue' networks showed consistent negative associations with GDM at both study visits, whereas the 'yellow' and 'brown' network showed positive associations with GDM risk at visit 0 among women who developed GDM but did not require medical intervention (online supplemental table S2). No association was observed among women who developed GDM and required intervention using insulin or other medication.

\section{DISCUSSION}

In this prospective study of 420 untargeted lipid metabolites in the plasma of multirace/ethnic US women in early pregnancy to mid-pregnancy, we observed that several plasma glycerolipids, some phospholipids and sterol lipids and SMs in early pregnancy both individually and interactively in distinct lipid networks were associated with subsequent risk of developing GDM. Overall, these GDM-related lipid metabolites and lipid networks 
also showed moderate to weak but consistent correlations with maternal glycemic biomarkers in early pregnancy to mid-pregnancy. Our results also suggested that acyl carbon chain length, double bond characteristics of the lipid metabolites may play an important role in maternal cardiometabolic risk.

Previous studies on maternal plasma lipidomics and GDM are sparse. We are aware of only two studies that have prospectively examined the association among predominantly Caucasian white ${ }^{13}$ or Han Chinese populations. ${ }^{12}$ Unlike our study, those two studies have implemented guidelines proposed by the International Association of Diabetes and Pregnancy Study Groups (IADPSG), which adopted a more stringent criterion for GDM diagnosis ${ }^{28}$ and in most populations lead to a significant increase in the number of women labeled as GDM. ${ }^{29}$ Hence, IADPSG criteria may potentially lead to classifying some women as having GDM who would be considered as having normal glucose tolerance by other diagnostic criteria (ie, Carpenter and Coustan criteria), and as such might reduce the variability in metabolite concentrations between GDM cases and non-GDM controls. Nonetheless, our findings are in consistent with previous prospective studies in other populations. In the UK study among predominantly white Caucasians, higher concentrations of glycerolipids, particularly TG (48:1), TG (51:1) and low-saturated phospholipid, PC (32:1) in the plasma in early second trimester have been associated with higher GDM risk. ${ }^{13}$ These findings are also in consistent with reports in non-pregnant population where glycerolipids, such as DGs and short, saturated/ low unsaturated TGs have been positively associated with insulin resistance, T2D and cardiovascular diseases. ${ }^{8} 27$ Cellular accumulation of DG, which is an intermediate of TG metabolism, ${ }^{30}$ has been associated with obesity and insulin resistance, ${ }^{31} 32$ mediated via protein kinase $\mathrm{C}$ activation. ${ }^{33}$ TGs and elevated free fatty acids have also been implicated in insulin resistance. ${ }^{30}$ Free fatty acids can generate oxidative stress, ${ }^{34}$ activating protein kinase $\mathrm{C}$ and thereby, contribute to insulin resistance. ${ }^{35}$ Our observed positive associations of several glycerolipids with GDM and maternal fasting and non-fasting glycemic biomarkers thus, reinforce existing literature on the etiological role of glycerolipids in the development of GDM.

In contrast, phospholipids rich with unsaturated fatty acids have been negatively associated with GDM. ${ }^{12} 3637$ In Han Chinese population, women who subsequently developed GDM had a lower concentration of several polyunsaturated PCs and PEs in the first trimester plasma. ${ }^{12}$ PCs rich with polyunsaturated fatty acids have also been negatively correlated with postload glucose, HbAlc, homeostatic model assessment of insulin resistance (HOMA-IR) and type 2 diabetes in non-pregnant population. ${ }^{38}$ In this study, we observed marked heterogeneity in associations between PCs and GDM based on the type of acyl carbon double bond to the glycerol backbone, where higher plasma concentrations of PCs with ester-linkage or ether-linkage were associated with lower risk of GDM. This pattern have also been reported in non-pregnant populations, where higher plasmenylphospholipids consisting of $O$-alk-1'-enyl linkage (also known as plasmalogen) have been associated with higher insulin sensitivity, lower insulin secretion and higher risk of type 2 diabetes. ${ }^{39}{ }^{40}$ Plasmalogen is an essential constituent of animal lipid membrane ${ }^{41}$ and act as an antioxidant to prevent lipoprotein oxidation. ${ }^{39}$ They also have anti-apoptotic and anti-inflammatory property, ${ }^{42}$ which may play a role in minimizing the risk for GDM.

SMs are structurally similar to PCs but contain ceramides instead of diacylglycerols. ${ }^{43}$ SMs are involved in plasma membrane signal transduction, cholesterol efflux and intracellular lipid and protein trafficking. ${ }^{44}$ Similar to our study, prior studies have also reported negative correlations between SMs and maternal fasting and 2 hour postload glucose, fasting insulin and HOMA-IR among women with a history of GDM..$^{10}$ These findings are reinforced by evidence in non-pregnant population where lower concentrations of SMs have been associated with higher HOMA-IR, homeostatic model assessment of $\beta$-cell function (HOMA-beta), fasting insulin and higher risk of type 2 diabetes ${ }^{40}$ and transitioning to type 2 diabetes from GDM. ${ }^{46}$ Animal studies have demonstrated that downregulation of sphingolipid metabolism in mouse islets and pancreatic beta-cell like cell lines have been associated with impaired glucose-stimulated insulin secretion without considerably impacting whole-body insulin sensitivity or glucose homeostasis. ${ }^{46}$

Similar to phospholipids and sphingolipids, our study demonstrated that higher concentrations of CEs in early pregnancy to mid-pregnancy were associated with lower risk of GDM. CEs are long-chain fatty acids linked to hydroxyl group of cholesterol, where plasma CEs tend to contain a relatively high proportion of polyunsaturated fatty acid. ${ }^{47}$ There are sparse data on CEs with GDM risk in pregnant population, although higher concentrations of CEs have been associated with lower risk of type 2 diabetes and cardiovascular diseases in non-pregnant populations, ${ }^{826}$ suggesting an overall beneficial effect of CEs on cardiometabolic health.

Our results suggest that GDM severity status may influence the associations between lipidomics and GDM risk as early as in early pregnancy. Women who were identified having severe disease requiring intervention using insulin or other medications at the time of diagnosis might have developed subclinical disease or pre-existing metabolic abnormality in early pregnancy, which could dysregulate lipid metabolites prior to the diagnosis of GDM. Furthermore, the therapeutic effect of GDM intervention could also influence lipidomics-GDM associations. The diminished difference in plasma concentrations of GDM-related metabolites between cases and controls could presumably be attributable to GDM treatment effect, which further suggests potential etiological role of lipid metabolites in GDM. Given the notable changes in concentrations of GDMrelated lipid metabolites across gestation as observed 
herein, and potential influence of the therapeutic effect of GDM intervention after the diagnosis, it is important to investigate the time-specific associations prior to GDM screening.

Our study has several unique strengths. We analyzed a comprehensive spectrum of lipid metabolites in relation to GDM risk in a multiracial/ethnic population as opposed to previous prospective studies ${ }^{12} 13$ that were conducted on a single race/ethnic group. Our prospective and longitudinal collection of lipidomics data allowed us to examine the temporal precedence in lipidomics and GDM associations. In particular, the case-control differences in most GDM-related lipid metabolites did not persist after the average gestational age at GDM diagnosis (ie, approximately week 27 ), which highlights the importance of temporal precedence in investigating the etiological roles of lipid metabolites. Furthermore, we had the unique ability to profile the longitudinal trends of lipid metabolites across pregnancy, which demonstrated differential temporal variations of the metabolites between women with and without GDM. We collected comprehensive data on maternal demographic and medical history to account for major GDM risk factors in our analysis. The overall consistent associations at both study visits suggest that our observed findings are internally replicable. In addition, we implemented a novel network analysis approach to investigate how metabolites within intertwined networks impact GDM risk via comprehensive interactions. ${ }^{21}$ Lastly, we analyzed a comprehensive panel of glycemic biomarkers with lipid metabolites to shed lights on the role of lipid metabolites into GDM etiology.

Some potential limitations of our study merit discussion. Plasma lipidome was measured using untargeted approach, which merits validation in another study and possibly using targeted approach. In this study, the plasma samples at 10-14 weeks were obtained in nonfasting state without controlling for mealtime and meal content. Hence, additional variability in lipid measurements might have introduced that was not related to variation in OGTT results at 28 weeks of gestation, thus reducing study power. However, this additional variability was likely small compared with the overall variability as within-subject variability in lipidomic profiles attributed to mealtime $(\approx 7 \%)$ were much smaller than the withinsubject variability not attributed to mealtime $(\approx 31 \%)$ or the between-subject variability $(\approx 62 \%),{ }^{48}$ albeit in a population less diverse in race/ethnicity than ours. Although this is the first study of its kind conducted in a race/ethnically diverse pregnant population, due to small sample size, we did not conduct stratified analysis by race/ ethnicity to examine the effect of ethnicity on the lipidome. Finally, the generalization of our study findings to the overall US pregnant population remains to be established, as the NICHD Fetal Growth Study enrolled pregnant women with low-risk prenatal profiles without major pre-existing chronic conditions. However, inclusion of overall healthy women may minimize reverse causality and residual confounding due to pre-existing complications and unhealthy lifestyle factors.

In this prospective study among pregnant women of multirace/ethnic groups with longitudinal measurement of plasma lipidome across pregnancy, we report that early pregnancy plasma concentrations of a number of glycerolipids and some phospholipid and sterol lipids and sphingomyelins both individually and interactively in distinct networks were prospectively associated with subsequent GDM risk. These GDM-related lipid metabolites and lipid networks showed consistent correlation pattern with maternal fasting and non-fasting glycemic biomarkers. If confirmed, these finding shed light on potential role of lipid metabolites as etiological biomarkers of GDM.

\section{Author affiliations}

${ }^{1}$ Department of Population Medicine and Harvard Pilgrim Health Care Institute, Harvard Medical School, Boston, Massachusetts, USA

${ }^{2}$ Epidemiology Branch, Division of Intramural Population Health Research, Eunice Kennedy Shriver National Institute of Child Health and Human Development, Bethesda, Maryland, USA

${ }^{3}$ Massachusetts General Hospital Center for Genomic Medicine, Boston,

Massachusetts, USA

${ }^{4}$ Program in Medical and Population Genetics, Broad Institute Harvard, Cambridge, Massachusetts, USA

${ }^{5}$ West Coast Metabolomics Center, University of California Davis, Davis, California, USA

${ }^{6}$ Division of Cancer Epidemiology and Genetics, National Cancer Institute, Bethesda, Maryland, USA

${ }^{7}$ Laboratory Medicine and Pathology, University of Minnesota System, Minneapolis, Minnesota, USA

${ }^{8}$ Division of Research, Kaiser Permanente Northern California, Oakland, California, USA

${ }^{9}$ Department of Population, Family, and Reproductive Health, Johns Hopkins University Bloomberg School of Public Health, Baltimore, Maryland, USA

${ }^{10}$ Department of Biostatistics, Harvard University T H Chan School of Public Health, Boston, Massachusetts, USA

Acknowledgements The authors would like to thank all the participants and staff at all participating clinical centers in the Eunice Kennedy Shriver National Institute of Child Health and Human Development (NICHD) Fetal Growth StudiesSingleton Cohort, including Christina Care Health Systems; University of California, Irvine; Long Beach Memorial Medical Center; Northwestern University; Medical University of South Carolina; Columbia University; New York Hospital Queens; St Peters' University Hospital; University of Alabama at Birmingham; Women \& Infants Hospital of Rhode Island; Fountain Valley Regional Hospital and Medical Center and Tufts University. The authors would also like to thank the C-TASC Corporation that provided data coordination and the NIH West Coast Metabolomics Center, UC Davis Genome Center, Davis, California that provided laboratory resources essential for biochemical analysis funded by NIH ESO30158 (to OF).

Contributors $\mathrm{CZ}$ designed the study and supervised data collection. MLR and Y-CAF analyzed data. MLR wrote the manuscript. LL, MLR and Y-CAF prepared the analysis plan. OF led laboratory testing, contributed to data interpretation. All authors contributed to the interpretation of the results and revision of the manuscript for important intellectual content and approved the final version of the manuscript. MLR and CZ are the guarantors of this work and, as such, had full access to all the data in the study and take responsibility for the integrity of the data.

Funding This research was supported by the Eunice Kennedy Shriver National Institute of Child Health and Human Development intramural funding (contract numbers HHSN275200800013C, HHSN275200800002I, HHSN27500006, HHSN275200800003IC, HHSN275200800014C, HHSN275200800012C, HHSN275200800028C, HHSN275201000009C and HHSN275201000001Z).

Competing interests None declared.

Patient consent for publication Not required. 
Ethics approval The study was approved by the institutional review boards of NICHD and all participating clinical sites, namely the Columbia University (New York), New York Hospital, Queens (New York), Christiana Care Health System (Delaware), Saint Peter's University Hospital (New Jersey), Medical University of South Carolina (South Carolina), University of Alabama (Alabama), Northwestern University (Illinois), Long Beach Memorial Medical Center (California), University of California, Irvine (California), Fountain Valley Hospital (California), Women and Infants Hospital of Rhode Island (Rhode Island) and Tufts University (Massachusetts). The IRB approval number is NICHD (09-CH-N152). All participants provided written informed consent before data collection.

Provenance and peer review Not commissioned; externally peer reviewed.

Data availability statement All data relevant to the study are included in the article or uploaded as supplemental information.

Supplemental material This content has been supplied by the author(s). It has not been vetted by BMJ Publishing Group Limited (BMJ) and may not have been peer-reviewed. Any opinions or recommendations discussed are solely those of the author(s) and are not endorsed by BMJ. BMJ disclaims all liability and responsibility arising from any reliance placed on the content. Where the content includes any translated material, BMJ does not warrant the accuracy and reliability of the translations (including but not limited to local regulations, clinical guidelines, terminology, drug names and drug dosages), and is not responsible for any error and/or omissions arising from translation and adaptation or otherwise.

Open access This is an open access article distributed in accordance with the Creative Commons Attribution 4.0 Unported (CC BY 4.0) license, which permits others to copy, redistribute, remix, transform and build upon this work for any purpose, provided the original work is properly cited, a link to the licence is given, and indication of whether changes were made. See: https://creativecommons.org/ licenses/by/4.0/.

ORCID iD

Mohammad L Rahman http://orcid.org/0000-0001-8322-0495

\section{REFERENCES}

1 Bellamy L, Casas J-P, Hingorani AD, et al. Type 2 diabetes mellitus after gestational diabetes: a systematic review and meta-analysis. Lancet 2009;373:1773-9.

2 American Diabetes Association. Gestational diabetes mellitus. Diabetes Care 2004;27 Suppl 1:S88-90.

3 Li S, Zhu Y, Yeung E, et al. Offspring risk of obesity in childhood, adolescence and adulthood in relation to gestational diabetes mellitus: a sex-specific association. Int J Epidemiol 2017:46:1533-41.

4 Zhu Y, Zhang C. Prevalence of gestational diabetes and risk of progression to type 2 diabetes: a global perspective. Curr Diab Rep 2016:16:7.

5 Mooradian AD. Dyslipidemia in type 2 diabetes mellitus. Nat Clin Pract Endocrinol Metab 2009;5:150-9.

6 Hivert MF, Perng W, Watkins SM, et al. Metabolomics in the developmental origins of obesity and its cardiometabolic consequences. J Dev Orig Health Dis 2015;6:65-78.

7 Cajka T, Fiehn O. Comprehensive analysis of lipids in biological systems by liquid chromatography-mass spectrometry. Trends Analyt Chem 2014:61:192-206.

8 Rhee EP, Cheng S, Larson MG, et al. Lipid profiling identifies a triacylglycerol signature of insulin resistance and improves diabetes prediction in humans. J Clin Invest 2011;121:1402-11.

9 Zhao C, Mao J, Ai J, et al. Integrated lipidomics and transcriptomic analysis of peripheral blood reveals significantly enriched pathways in type 2 diabetes mellitus. BMC Med Genomics 2013;6 Suppl 1:S12.

10 Allalou A, Nalla A, Prentice KJ, et al. A predictive metabolic signature for the transition from gestational diabetes mellitus to type 2 diabetes. Diabetes 2016;65:2529-39.

11 Lappas M, Mundra PA, Wong G, et al. The prediction of type 2 diabetes in women with previous gestational diabetes mellitus using lipidomics. Diabetologia 2015;58:1436-42.

12 Law KP, Mao X, Han T-L, et al. Unsaturated plasma phospholipids are consistently lower in the patients diagnosed with gestational diabetes mellitus throughout pregnancy: a longitudinal metabolomics study of Chinese pregnant women Part 1. Clin Chim Acta 2017;465:53-71.

13 Lu L, Koulman A, Petry CJ, et al. An unbiased lipidomics approach identifies early second trimester lipids predictive of maternal glycemic traits and gestational diabetes mellitus. Diabetes Care 2016;39:2232-9.

14 Grewal J, Grantz KL, Zhang C, et al. Cohort profile: NICHD fetal growth Studies-Singletons and twins. Int J Epidemiol 2018;47:25-25I.

15 Committee on Practice Bulletins-Obstetrics. Practice Bulletin No. 137: gestational diabetes mellitus. Obstet Gynecol 2013;122:406-16.

16 American Diabetes Association. (2) classification and diagnosis of diabetes. Diabetes Care 2015;38 Suppl:S8-16.

17 Barupal DK, Fan S, Wancewicz B, et al. Generation and quality control of lipidomics data for the alzheimer's disease neuroimaging initiative cohort. Sci Data 2018;5:180263.

18 Barupal DK, Fan S, Wancewicz B, et al. Generation and quality control of lipidomics data for the Alzheimer's disease neuroimaging initiative cohort. Sci Data 2018;5:180263.

19 Cajka T, Smilowitz JT, Fiehn O. Validating quantitative untargeted lipidomics across nine liquid Chromatography-High-Resolution mass spectrometry platforms. Anal Chem 2017;89:12360-8.

20 Hernán MA, Hernández-Díaz S, Werler MM, et al. Causal knowledge as a prerequisite for confounding evaluation: an application to birth defects epidemiology. Am J Epidemiol 2002;155:176-84.

21 Langfelder P, Horvath S. WGCNA: an R package for weighted correlation network analysis. BMC Bioinformatics 2008;9:559.

22 SSP S. Computational discrete mathematics: Combinatorics and graph theory with Mathematica. 1st Edition ed, 1990.

23 Yip AM, Horvath S. Gene network interconnectedness and the generalized topological overlap measure. BMC Bioinformatics 2007;8:22.

24 Langfelder P, Zhang B, Horvath S. Defining clusters from a hierarchical cluster tree: the dynamic tree cut package for $\mathrm{R}$. Bioinformatics 2008;24:719-20.

25 Bareinboim E, Barbosa VC. Descents and nodal load in scale-free networks. Phys Rev E Stat Nonlin Soft Matter Phys 2008;77:046111.

26 Toledo E, Wang DD, Ruiz-Canela M, et al. Plasma lipidomic profiles and cardiovascular events in a randomized intervention trial with the Mediterranean diet. Am J Clin Nutr 2017;106:973-83.

27 Razquin C, Liang L, Toledo E, et al. Plasma lipidome patterns associated with cardiovascular risk in the PREDIMED trial: a casecohort study. Int J Cardiol 2018:253:126-32.

28 Metzger BE, Gabbe SG, Persson B, et al. International association of diabetes and pregnancy study groups recommendations on the diagnosis and classification of hyperglycemia in pregnancy: response to Weinert. Diabetes Care 2010;33:e98-82.

29 Jenum AK, Mørkrid K, Sletner L, et al. Impact of ethnicity on gestational diabetes identified with the who and the modified international association of diabetes and pregnancy study groups criteria: a population-based cohort study. Eur J Endocrinol 2012;166:317-24.

30 Boden G, Laakso M. Lipids and glucose in type 2 diabetes: what is the cause and effect? Diabetes Care 2004;27:2253-9.

31 Itani SI, Ruderman NB, Schmieder F, et al. Lipid-induced insulin resistance in human muscle is associated with changes in diacylglycerol, protein kinase C, and lkappaB-alpha. Diabetes 2002;51:2005-11.

32 Chibalin AV, Leng Y, Vieira E, et al. Downregulation of diacylglycerol kinase delta contributes to hyperglycemia-induced insulin resistance. Cell 2008;132:375-86.

33 Badin P-M, Vila IK, Louche K, et al. High-fat diet-mediated lipotoxicity and insulin resistance is related to impaired lipase expression in mouse skeletal muscle. Endocrinology 2013;154:1444-53

34 Ceriello A. Oxidative stress and glycemic regulation. Metabolism 2000;49:27-9.

35 Griffin ME, Marcucci MJ, Cline GW, et al. Free fatty acid-induced insulin resistance is associated with activation of protein kinase $\mathrm{C}$ theta and alterations in the insulin signaling cascade. Diabetes 1999;48:1270-4.

36 Dudzik D, Zorawski M, Skotnicki M, et al. Metabolic fingerprint of gestational diabetes mellitus. J Proteomics 2014;103:57-71.

37 Lehmann R, Friedrich T, Krebiehl G, et al. Metabolic profiles during an oral glucose tolerance test in pregnant women with and without gestational diabetes. Exp Clin Endocrinol Diabetes 2015;123:483-438.

38 Floegel A, Stefan N, Yu Z, et al. Identification of serum metabolites associated with risk of type 2 diabetes using a targeted metabolomic approach. Diabetes 2013;62:639-48.

39 Wallner S, Schmitz G. Plasmalogens the neglected regulatory and scavenging lipid species. Chem Phys Lipids 2011;164:573-89.

40 Razquin C, Toledo E, Clish CB, et al. Plasma lipidomic profiling and risk of type 2 diabetes in the PREDIMED trial. Diabetes Care 2018:41:2617-24. 
41 Magnusson CD, Haraldsson GG. Ether lipids. Chem Phys Lipids 2011;164:315-40.

42 Huynh K, Martins RN, Meikle PJ. Lipidomic Profiles in Diabetes and Dementia. J Alzheimers Dis 2017:59:433-44.

43 Merrill AH, Schmelz EM, Dillehay DL, et al. Sphingolipids--the enigmatic lipid class: biochemistry, physiology, and pathophysiology. Toxicol Appl Pharmacol 1997;142:208-25.

44 Brown DA, London E. Structure and origin of ordered lipid domains in biological membranes. J Membr Biol 1998;164:103-14.

45 Lemaitre RN, Yu C, Hoofnagle A, et al. Circulating sphingolipids, insulin, HOMA-IR, and HOMA-B: the strong heart family study. Diabetes 2018;67:1663-72.
46 Khan SR, Mohan H, Liu Y, et al. The discovery of novel predictive biomarkers and early-stage pathophysiology for the transition from gestational diabetes to type 2 diabetes. Diabetologia 2019;62:687-703.

47 Christie WW, Hunter ML, Moore JH, et al. Effect of diet on the composition of cholesteryl esters of sheep adrenals. Lipids 1975;10:649-51.

48 Begum H, Li B, Shui G, et al. Discovering and validating betweensubject variations in plasma lipids in healthy subjects. Sci Rep 2016;6:19139. 